\title{
THE LINES AND PLANES CONNECTING THE POINTS OF A FINITE SET
}

\author{
BY \\ TH. MOTZKIN
}

0.1. Not later than 1933 I made the following conjecture, originally in the form of a statement on the minors of a matrix $\left({ }^{1}\right)$.

$\mathrm{T}_{d}$. Any $n$ points in $d$-space that are not on nne hyperplane determine at least $n$ connecting hyperplanes.

$\mathrm{T}_{1}$ is trivial. It is easy to see (4.3) that $\mathrm{T}_{d}$ is a consequence of $\mathrm{T}_{d-1}$ and

$\mathrm{U}_{d}$. Any $n$ points in $d$-space that are not on one hyperplane determine at least one ordinary hyperplane, that is, a connecting hyperplane on which all but one of the given points are on one linear $(d-2)$-space.

In particular, $\mathrm{T}_{2}: n$ not collinear points are connected by at least $n$ straight lines, is true if $\mathrm{U}_{2}$ holds: for $n$ not collinear points there is a straight line through only two of them. Now the nine inflexions of a plane cubic show that $\mathrm{U}_{2}$ does not hold in the complex plane. Nevertheless $H$. Hanani gave in 1938 a combinatorial proof of $T_{2}$ for every (not only the real) projective plane. A greatly simplified version of this proof is given in 4.4. In $1939 \mathrm{~A}$. Robinson proved $\mathrm{U}_{2}$ for the real plane; in $1943 \mathrm{I}$ found another very short proof (respectively the second and first proof in 1.1)( ${ }^{2}$ ). In 1944 I proved $\mathrm{U}_{3}$ for real 3-space (1.4 and 1.5). $\mathrm{U}_{d}$ is still unproved.

The existence of three nonconcurrent ordinary lines is proved in 2.2 , of four ordinary planes in 2.3. In $\S 3$ those $n$ are determined for which $\mathrm{U}_{2}$ holds for every field of coordinates $F ; 3.6$ contains conditions for the fields $F$ for which $\mathrm{U}_{2}$ holds for every $n$. An application to configurations is made in $\S 5$.

Recently I learned that $P$. Erdös had dealt with $U_{2}$ (for the real plane) and $T_{2} . U_{2}$ had been conjectured by Sylvester (1893) and by Erdös (1933) and proved by T. Gallai (1933) and others (my proof being substantially that of R. Steinberg (1944)) ( $\left.{ }^{3}\right)$. $T_{2}$ had been proved by G. Szekeres (1940 or 1941) and by N. G. de Bruijn and Erdös $\left({ }^{4}\right)$. A combination of part

Presented to the Society, September 2, 1949; received by the editors June 26, 1950.

(1) Beiträge zur Theorie der linearen Ungleichungen, Dissertation, Basel, Jerusalem, 1936, p. 31 .

(2) It is easy to give a finite turn to Robinson's proof: consider the smallest (in area) triangle with a line through vertex and base, and so forth.

(3) References in H. S. M. Coxeter, The real projective plane, New York, 1949, p. 26. Remark (Nov. 1950): L. M. Kelly's proof by a point and connecting line with minimal distance (it follows that the line is ordinary) holds also on the sphere and for the dual.

(4) On a combinatorial problem, Neder. Akad. Wetensch. vol. 10 (1948) pp. 421-423. Their question whether the number of ordinary lines tends to infinity with $n$ is answered in the affirmative in 2.4 . 
of the latter proof with a combinatorial lemma (4.6) enabled me to prove $\mathrm{T}_{d}(4.5)(5)$.

0.2 . We consider $n$ different points $P_{i}, i=1, \cdots, n$, in the projective $d$-dimensional space or briefly $d$-space, and their connecting lines $p_{k}$, connecting planes $\pi_{m}$, and connecting hyperplanes, whose number is at most $C_{n, 2}, C_{n, 3}$ and $C_{n, d}$. As usual, connecting lines, meeting-points, and so on, are denoted by juxtaposition: $P Q, p q, p \cdot q$ (meeting point), $\pi \cdot q$, and so on.

1. Existence of an ordinary line or plane.

1.1. If $n$ points in the real projective plane are not on one straight line, then there exists a straight line containing exactly two of the points.

Such a straight line is called an ordinary line.

Proof. Choose a straight line $q$ that contains $P_{1}$ and no other $P_{i}$ or $p_{k} \cdot p_{l}$. Let $P_{1} Q, Q=q \cdot p_{1}$, be a segment on $q$ not met by any $p_{k}$, and $P_{2} P_{3}$ a segment on $p_{1}$ that contains $Q$ and no $P_{i}$. Either $p_{1}$ is ordinary, or $p_{1}$ contains a point $P_{4}$. Then $P_{1} P_{4}$ is ordinary: for if it contained $P_{5}$ then $P_{2} P_{5}$ or $P_{3} P_{5}$ would meet the segment $P_{1} Q$.

Alternatively: proof of the dual theorem. Let $P_{1}, P_{2}, P_{3}$ be nonconcurrent straight lines. Either $P_{2} \cdot P_{3}$ is ordinary, or on a line $P_{4}$. Choose the line at infinity so that $P_{1}, P_{2}, P_{4}$ meet in finite points and that the point $P_{1} \cdot P_{3}$ is between $P_{1} \cdot P_{2}$ and $P_{1} \cdot P_{4}$. Either $P_{1} \cdot P_{3}$ is ordinary, or on a line $P_{5} ; P_{5}$ divides either the triangle $\triangle P_{1} P_{3} P_{2}$ or $\triangle P_{1} P_{3} P_{4}$, say $\triangle P_{1} P_{2} P_{3}$. Either $P_{2} \cdot P_{5}$ is ordinary, or on a line $P_{6}$, which divides either $\triangle P_{2} P_{5} P_{1}$ or $\triangle P_{2} P_{5} P_{3}$. If this could be repeated ad inf., then the number of triangles, and of lines $P_{i}$, would be infinite.

The existence of an ordinary line would also follow from that of an ordinary point, that is, a point on at least $n / 2$ connecting lines. The existence of such a point has not been proved or disproved.

1.2. Invalid analogue in 3-space. Consider the Desargues configuration of ten meeting-points of five planes $\alpha_{1}, \cdots, \alpha_{5}$ in general position. Among any three of these points there are necessarily two that have two planes in common, so that a plane through them must contain the third given point on their connecting line. Hence there is no connecting plane containing exactly three of the points. There exist planes through three points only, but these points are collinear.

Now consider $n_{1} \geqq 2$ on one and $n_{2} \geqq 2$ points on the other of two skew straight lines. Here every connecting plane contains at least $\min \left(n_{1}, n_{2}\right)+1$ of the given points, and also every plane through more than two of the points contains at least $\min \left(n_{1}, n_{2}\right)$ points. Hence 1.1 cannot be extended to planes in 3-space by replacing "two" by "three" or "between 3 and $k$. "

I know of no other examples with no connecting plane containing exactly three of the points.

(5) This and some other results were announced in an abstract of the present paper published in Bull. Amer. Math. Soc. Abstract 55-11-573. 
1.3. Valid analogues.

(1) If $n$ points $P_{i}$ in real projective 3-space are not on one plane, then there exists a connecting plane all of whose points $P_{i}$ are on two straight lines through one of them, $P_{1}$, where $P_{1}$ may be chosen beforehand.

This follows at once from 1.1. For project, from $P_{1}$, the other points $P_{i}$ on a plane. In this plane there is a straight line $l$ through exactly two of the projections. The plane $P_{1} l$ fulfills the condition. (The projection must be made from a point $P_{i}$, for otherwise the plane obtained need not be a connecting plane.)

(2) If $n$ points in the real affine plane are not on one straight line or circle, then there is a straight line or circle containing exactly three of the points; one of the three points may, moreover, be chosen beforehand.

For apply an inversion at the chosen point and 1.1 .

There follows:

If $n$ points on the real sphere are not on one plane, then there is a plane containing exactly three of them.

By a projective transformation the same holds for any quadric without straight lines. On ruled quadrics the proposition fails, by 1.2, since they contain pairs of skew lines.

The theorem is also an immediate consequence of (1) and holds for every convex surface that contains no straight segment.

1.4. In 1.5 , we need the following lemma.

If $T=\triangle P_{1} P_{2} P_{3}$ is a closed triangle in the real projective plane, and if $P_{4}, \cdots, P_{n}, n>3$, are points outside $T$, then at least one of the following statements is true:

(1) Some connecting line $p_{k}$ is outside $T$;

(2) Some $p_{k}$ has exactly one point in common with $T$ and contains exactly two points $P_{i}$;

(3) $n=6$, and $T$ is part of, and inscribed in, $\triangle P_{4} P_{5} P_{6}$.

Proof. Make the plane affine by choosing a line at infinity outside $T$ and through none of the points $p_{i}$. Let $C$ be the smallest convex polygon containing all $P_{i}$. Any side of $C$ that contains no vertex of $T$ complies with (1). If every side of $C$ contains a vertex of $T$, then $C$ and $T$ are situated as indicated in one of the following figures:

1.

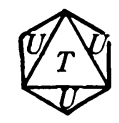

2.

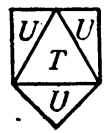

3.

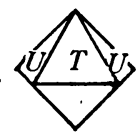

4.

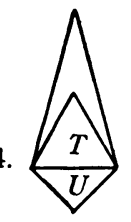

5.

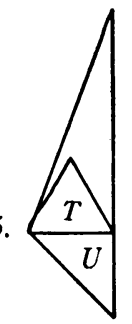

6.

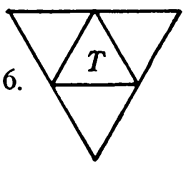


in which every triangular part denoted by $U$ may also be missing.

Now in every triangle $U=\triangle P_{1} P_{2} P_{4}$ there is a side, say the segment $P_{1} P_{4}$, whose continuation does not belong to $C$. Then either $P_{1} P_{4}$ complies with (2), or the segment $P_{1} P_{4}$ contains a point $P_{5}$. Possibly $\triangle P_{1} P_{2} P_{5}$ contains a point $P_{6}$, and so on; at any rate we must, the number of points $P_{i}$ being finite, at last arrive at a point $P_{m}$ such that $\triangle P_{1} P_{2} P_{m}$ contains no $P_{i}$. If neither $P_{1} P_{m}$ nor $P_{2} P_{m}$ complies with (2), $P_{m}$ will be on segments $P_{1} P_{j}$ and $P_{2} P_{k}$, and $P_{j} P_{k}$ complies with (1).

For case $6, n>6$, proceed similarly.

In case 4 or 5 without $U$, with $C=\triangle P_{1} P_{2} P_{4}$, choose a new line at infinity near $P_{4}$ between $P_{4}$ and the segment $P_{1} P_{2}$, which reduces this case to one of the others, including case 6 .

1.5. The true, nontrivial, analogue of 1.1 in 3 -space is:

If $n$ points $P_{i}$ in real projective 3 -space are not on one plane, then there exists a connecting plane such that all $P_{i}$ but one on it are collinear.

Such a connecting plane is called an ordinary plane.

Proof. Choose a straight line $q$ that contains $P_{1}$ and no other point of any $\pi_{l} \cdot \pi_{m}$. Let the segment $P_{1} Q, Q=q \cdot \pi_{1}$, be a segment on $q$ not met by any $\pi_{m}$, and $T=\triangle P_{2} P_{3} P_{4}$ a triangle on $\pi_{1}$ that contains $Q$ and no $P_{i}$. (Such a triangle is easily obtained: a triangle $\triangle P_{j} P_{k} P_{l}$ containing $Q$ and some $P_{i}$ is divided into triangles by segments connecting the $P_{i}$ and the vertices, and the division is repeated as long as necessary.)

Now choose $\pi_{1}$ as the plane at infinity and $P_{1} P_{2}, P_{1} P_{3}, P_{1} P_{4}$ as axes for affine coordinates, oriented so that the coordinates of a point of the segment $P_{1} Q$ are positive. Then every coordinate of every finite $P_{i}$ is nonpositive; otherwise one of $P_{i} P_{2} P_{3}, P_{i} P_{3} P_{4}, P_{i} P_{4} P_{2}$ would meet the segment $P_{1} Q$.

Hence if $\pi_{1}$ contains a connecting line $p_{k}$ outside $T$, then $P_{1} p_{k}$ is a plane as required. The same is true if $p_{k}$ has exactly one point in common with $T$ and contains exactly two points $P_{i}$. If $T$ is part of, and inscribed in, $\triangle P_{5} P_{6} P_{7}$, let $T_{1}=\triangle P_{2} P_{5} P_{3}, T_{2}=\triangle P_{2} P_{5} P_{4}$. For $Q$ in $T_{1}$, take $T_{1}$ instead of $T$ and $p_{k}=P_{2} P_{4} ;$ for $T_{2}$, take $p_{k}=P_{2} P_{3}$. Hence in every case of $1.4,1.5$ is proved; if $\pi_{1}$ contains no $P_{i}, i>4$, then $\pi_{1}$ is itself a plane as required.

There follows the existence of an ordinary line for $n$ points in 3-space that are not on one straight line. But this follows already from 1.1, by projecting the $n$ points from a point $Q$ that is on no $\pi_{m}$. In the same way the existence of an ordinary line, or plane, is proved for $d$-space, $d>3$. The existence of an ordinary hyperplane remains still to be established.

\section{Existence of more than one ordinary line or plane.}

2.1. In every, not only the real, projective space of any number of dimensions we have immediately:

If every connecting line of $n$ points $P_{i}$ contains at least one of the points $Q_{1}, \cdots, Q_{m}$, and if the points $P_{1}, \cdots, P_{r}$ that are different from the points $Q_{j}$ are not on one straight line, then $r \leqq(m-1)^{2}$. 
Indeed, through either of $P_{1}$ and $P_{2}$ there are at most $m-1$ lines $p_{k}$ besides $P_{1} P_{2}$; these lines meet in at most $(m-1)(m-2)$ points $R_{l}$ other than $Q_{j}$. The points $R_{l}$ include every point $P_{i}, i=3, \cdots, r$, that is not on $P_{1} P_{2}$, and if such a $P_{i}$ exists then at most $m-1$ of $P_{1}, \cdots, P_{r}$ are on $P_{1} P_{2}$.

There is no obvious analogue for connecting planes.

If every connecting line of $n$ points $P_{i}$ contains one of the points $Q_{1}, Q_{2}$, then $n-1$ points $P_{i}$ are on one straight line.

This is a special case of the preceding statement. Its analogue for planes is as follows.

If every connecting plane of $n$ points $P_{i}$ contains one of the points $Q_{1}, Q_{2}$, then either all $P_{i}$ are on two straight lines or $n-1$ points $P_{i}$ are on one plane.

For if $P_{1}, \cdots, P_{4} \neq Q_{1}, Q_{2}$ are not on one plane, then we may suppose $Q_{1}$ on $P_{1} P_{2}, Q_{2}$ on $P_{3} P_{4}$. Any $P_{5} \neq Q_{1}, Q_{2}$ must be on $P_{1} P_{3} P_{2}$ or $P_{1} P_{3} P_{4}$, and on $P_{1} P_{4} P_{2}$ or $P_{1} P_{4} P_{3}$, that is, on the quadrangle $P_{1} P_{2} P_{3} P_{4}$; likewise on $P_{1} P_{2} P_{4} P_{3}$; hence on $P_{1} P_{2}$ or $P_{3} P_{4}$.

2.2. A finite number of straight lines in the real projective plane that do not all pass through one point form a proper division of the plane into polygons.

If $n$ points $P_{i}$ in the real projective plane are not on one straight line and if $P_{1}$ is on no ordinary line, then the lines $p_{k}$ that do not pass through $P_{1}$ form $a$ proper division of the plane. In this division the sides of the polygon containing $P_{1}$ are ordinary lines.

For if the division were not proper, then, by 2.1 , all $P_{i}$ but one would be on one straight line, in which case every $P_{i}$ is on an ordinary line. The second statement follows from the proof of 1.1 .

If $n$ points in the real projective plane are not on one straight line, then they determine at least three ordinary lines.

This follows from the preceding statement if there is a point $P_{1}$ that is on no ordinary line. But if every $P_{i}$ is on one of two ordinary lines $p_{1}$ and $p_{2}$, and if $P_{1} \neq p_{1} \cdot p_{2}$ is on $p_{1}$ and $P_{2} \neq p_{1} \cdot p_{2}$ is on $p_{2}$, then $P_{1} P_{2}$ is also an ordinary line.

There follow at once corresponding refinements of the statements in 1.3.

If $P_{2}, \cdots, P_{n}$ in the real projective plane are not on one straight line, then there exists an ordinary line not through $P_{1}$.

Again this follows from the last statement but one if there is a point $P_{i}$ that is on no ordinary line. But if every $P_{i}$ is on an ordinary line, and if every ordinary line passes through $P_{1}$, then $P_{2}, \cdots, P_{n}$ determine no ordinary line and are therefore collinear.

If no $n-1$ of $n$ given points in the real projective plane are collinear, then the ordinary lines determined by the $n$ points are not concurrent.

For if they were, add their common point to the given points and apply the preceding statement.

By inversion we have: 
If $n$ points in the real affine plane are not on one straight line or circle, then there is a circle containing exactly three of the points; one of the three points may, moreover, be chosen beforehand.

2.3. If $n$ points in the real projective plane, or $d$-space, are not on one straight line and if they determine not more than $m$ ordinary lines, then $n$ $\leqq C_{m+2,2}$.

For $d>2$, project on $(d-1)$-space from a point on no connecting plane; $n$ and $m$ remain unchanged.

For $d=2$, the ordinary lines divide the plane into at most $C_{m, 2}+1$ polygons. In each polygon there is at most one point $P_{i}$ (see proof of 2.2). Hence $n \leqq C_{m, 2}+1+2 m=C_{m+2,2}$.

Thus for sufficiently large $n$ the number of ordinary lines exceeds any given number $m$.

2.4. If $n$ points in the real projective plane determine exactly three ordinary lines, then $n$ equals $3,4,6$, or 7 .

Proof. If the three ordinary lines are concurrent, then, by $2.2, n-1$ points are collinear whence $n=4$.

If the ordinary lines form four triangles $T_{1}, \cdots, T_{4}$, and if $T_{1}$ contains a point $P_{i}$, then every connecting line that crosses $T_{1}$ passes through $P_{i}$. If $T_{1}$ contains $P_{1}$ and $T_{2}$ contains $P_{2}$, there can therefore be no $P_{i}$ on the sides of $T_{1}$ and $T_{2}$ except the common vertices and points of $P_{1} P_{2}$. Hence the common vertices are, say, $P_{5}$ and $P_{6}$. The lines $P_{1} P_{5}$, and so on, are not ordinary, hence every $T_{i}$ contains a point $P_{i}$; the last vertex is $P_{7}$. The points $P_{5}, P_{6}, P_{7}$ are the diagonal points of $P_{1}, \cdots, P_{4} ; n=7$.

If $T_{1}$ contains $P_{1}$, and no other $T_{i}$ a point $P_{i}$, then since every connecting line that crosses $T_{1}$ passes through $P_{1}$, and every connecting line through $P_{1}$ is not ordinary, we obtain easily that $n \leqq 7$. Hence the number $m$ of connecting lines through $P_{1}$ is 2 or 3 . If $m=2$, with $k \geqq 2$ and $l \geqq 2$ points besides $P_{1}$ on the connecting lines through $P_{1}$, then there are $k l \geqq 4$ ordinary lines. If $m=3$, with two points besides $P_{1}$ on each line, then there are easily seen to be two ordinary lines through one of those points; but if six points are on three ordinary lines, then each of them must be on only one ordinary line.

If no $T_{i}$ contains a point $P_{i}$, then either $n=6$ (the meeting-points of four straight lines) or $n=3$.

2.5. If $n$ points in real projective $d$-space, $d>2$, are not on one hyperplane, then they determine at least $C_{d+1,2}$ ordinary lines; in fact more if $n>d+1$.

Suppose this is true for $d-1$. Projecting from $P_{1}$ on a linear $(d-1)$ space we obtain points $Q_{i}$ not on one linear $(d-2)$-space. Every line $p_{j}$ projected into an ordinary line $Q_{1} Q_{2}$ is ordinary. If both, one, or neither of the projecting lines $P_{1} Q_{1}$ and $P_{1} Q_{2}$ is ordinary, then the number of these $p_{j}$ is respectively one, at least two, or at least four. Therefore the total number $m_{P}$ of ordinary lines $p_{j}$ surpasses that of ordinary lines $q_{k}$ at least by the number $e$ of points $Q_{i}$ situated on the ordinary $q_{k}$. Since there are at least $C_{d, 2}$ and at 
most $C_{e, 2}$ such $q_{k}$ we have $e \geqq d$. Hence $m_{P}$ can be exactly $C_{d, 2}+d$ only if the number of all $Q_{i}$ is $d$. But in this case every $P_{1} Q_{i}$ that is not ordinary causes an increase in $m_{P}$, whence $n$ must be $d+1$.

For $d=3$ the conclusions are obvious if every connecting line is ordinary. Otherwise take two connecting planes through a not ordinary line and a third connecting plane: they each carry at least three ordinary lines, and together, since only two meeting-lines can have been counted twice, at least seven ordinary lines.

That $m_{P} \geqq 6$ follows also from 2.7 (twelve lines less at most six meetinglines, or one triple meeting-line and three meeting-lines, or one quadruple meeting-line).

If we try to prove the case $d=3$ by the same argument as $d>3$, we have, by 2.4 , also to consider the possibility of seven points $Q_{i}$. But for $P_{2}$ and $P_{3}$ on $P_{1} Q_{4}$ and $P_{4}$ on $P_{1} Q_{5}$ either $P_{2} P_{4}$ or $P_{3} P_{4}$ would be ordinary.

Incidentally we see that this set of seven points is rigid, that is, it cannot be obtained from a set of points in 3-space that are not on one plane by projection from a point that is on no connecting plane; for such a projection leaves $m_{P}$ unchanged. A set of points all but one of which are collinear is also rigid; so is any set for which every two $p_{k}$ can be joined by a chain of adjacent triangles, that is, triangles with two common vertices and two common sides.

2.6. A finite number of planes in real projective 3 -space that do not pass through one point form a proper division of space into polyhedra.

If no $n-1$ of $n$ points $P_{i}$ in real projective 3-space are on one plane and if $P_{1}$ is on no ordinary plane, then the planes $\pi_{m}$ that do not pass through $P_{1}$ form a proper division of space. In this division the sides of the polyhedron containing $P_{1}$ are ordinary planes.

For if the division were not proper, then, by 2.1 , all $P_{i}$ would be on two straight lines, in which case every $P_{i}$ is on an ordinary plane. The second statement follows from the proof of 1.5 .

2.7. If $n$ points in real projective 3-space are not on one plane, then they determine at least four ordinary planes.

Proof. By 2.6 we may suppose that every $P_{i}$ is on an ordinary plane. (If $P_{2}, \cdots, P_{n}$ are on one plane and $n>4$ then there are at least four connecting planes through $P_{1}$.)

We now observe that if a point $P_{1}$ is on only one ordinary plane $\pi_{1}$, then there are, through $P_{1}$, three connecting lines that are not ordinary lines and not in one plane. (One of these lines may be in $\pi_{1}$.) For otherwise we would, by projecting from $P_{1}$ and applying 2.2, obtain another ordinary plane through $P_{1}$.

For $n$ points with only two ordinary planes this leads to a contradiction. Suppose, therefore, that there are exactly three ordinary planes $\pi_{1}, \pi_{2}, \pi_{3}$, and that for $k=1,2,3$ all points $P_{i}$ of $\pi_{k}$ except $P_{k}$ are on $p_{k}$. We then have to 
consider several cases.

(1) If a point $P_{i}$ is on all three planes, then projection from $P_{i}$ and application of 2.2 show easily that the above notations may be made in such a way that $P_{i}$ is on $p_{1}$ and $p_{2}$.

(2) If $P_{i}$ is on only $\pi_{1}$ and $\pi_{2}$, we see in the same manner that $p_{1}$ and $p_{2}$ pass through $P_{i}$ and are not ordinary lines. Hence cases (1) and (2) exclude each other.

Now by our first remark there are, in case (1), three not coplanar, not ordinary connecting lines through $P_{1}$. This implies that $P_{i}$ is also on $p_{3}$ and that $P_{1}, P_{2}, P_{3}$ are collinear. But then $P_{i} P_{1} P_{2}$ would be a fourth ordinary plane.

Applying the same remark, in case (2), to $P_{3}$ we see that $P_{3}$ is on the plane $p_{1} p_{2}$ and on the line $P_{1} P_{2}$. Now applying it to $P_{1}$ and to $P_{2}$ we find that $p_{3}$ is on $p_{1} p_{2}$, which is impossible.

Hence every $P_{i}$ may be supposed to be on only one ordinary plane.

(3) If $P_{1}, P_{2}, P_{3}$ are collinear, application of the said remark to these points shows that $P_{1}$ is on $p_{2} p_{3}, P_{2}$ on $p_{3} p_{1}, P_{3}$ on $p_{1} p_{2}$. Hence $p_{1}, p_{2}, p_{3}$ are concurrent. But then every plane connecting $P_{4}$ on $p_{1}$ with points $P_{i}$ on $p_{2}$ and $p_{3}$, except at most one plane, would be ordinary.

(4) Now application of the same remark to $P_{1}$ shows that $P_{1} P_{2}$ passes through a point $P_{3}{ }^{\prime}$ on $p_{3}$; similarly $P_{1}^{\prime}=P_{2} P_{3} \cdot p_{1}, P_{2}^{\prime}=P_{3} P_{1} \cdot p_{2}$. If there were only two points $P_{i}$ on $p_{3}$, then $P_{3}^{\prime}$ in place of $P_{3}$ in case (3) would lead to a contradiction. Applying the remark to the points $P_{i}$ of $p_{3}$, we see that they are only $P_{3}^{\prime}, P_{3}^{\prime \prime}$ (on $p_{1} P_{2} P_{3}$ ), $P_{3}^{\prime \prime \prime}$ (on $p_{2} P_{1} P_{3}$ ); similarly for $p_{1}$ and $p_{2}$. Since the line $P_{1} P_{2} P_{3}^{\prime}$ is coplanar with $P_{3}, P_{1}^{\prime}, P_{2}^{\prime}$ and with $P_{3}^{\prime \prime}, P_{3}^{\prime \prime \prime}, P_{2}^{\prime \prime}, P_{1}^{\prime \prime \prime}$, and since $P_{3}, P_{1}^{\prime \prime}, P_{2}^{\prime \prime \prime}$ are collinear, at least one of the planes $P_{1} P_{2} P_{1}^{\prime \prime}$ and $P_{1} P_{2} P_{2}^{\prime \prime \prime}$ would be ordinary.

2.8. Even more than in 2.4 it may be cumbersome to find the sets with exactly four ordinary planes, since it is not certain that $n$ is bounded for these sets. Again things are simpler for more dimensions.

If $n$ points in real projective 4-space are not on one hyperplane, then they determine at least ten ordinary planes; in fact more if $n>5$.

If there is a not ordinary plane $P_{1} P_{2} P_{3}$, let $P_{1}, \cdots, P_{5}$ be not on one hyperplane. Then the four 3 -spaces, other than $P_{2} P_{3} P_{4} P_{5}$, determined by these five points each carry at least four ordinary planes, and, since at most five meeting-planes are counted twice, together at least eleven ordinary planes.

If every connecting plane is ordinary, ten such planes are determined by any five points that are not on one hyperplane. A sixth point entails at least three more connecting planes.

3. Values of $n$ for which an ordinary line must exist in every projective plane.

3.1. For $n=7$, and for every $n \geqq 9$, there is a Desarguesian projective plane 
and in it a set of $n$ points $P_{i}$ not all of which are on one straight line, such that every connecting line contains at least three points $P_{i}$.

Proof. Consider the projective plane $A_{m}$ over a finite field with $m$ elements. Let $B_{m}$ be the set of all the points on three straight lines through a point $O$ of $A_{m}, C_{m}(m>2)$ the same set without $O, D_{m}(m>3)$ the set $C_{m}$ plus two points not on a straight line through $O$, and $E_{m}(m>2)$ the set $B_{m}$ plus an arbitrary set of points of which no straight line through $O$ contains exactly one. Obviously $A_{m}, \cdots, E_{m}$ are sets as required, with respectively $n=m^{2}+m+1, n=3 m+1, n=3 m, n=3 m+2,3 m+3 \leqq n \leqq m^{2}+m+1$.

Since for prime $m>6$ there is a prime $p$ with $m<p<2 m$, whence $3 p+3$ $<6 m+3<m^{2}+m+1$, the values of $n$ corresponding to $E_{m}$ with prime $m$ are 12,13 , and every $n \geqq 18$. We obtain $n=7$ by $A_{2}=B_{2}, 9$ by $C_{3}, 10$ by $B_{3}, 12$ by $C_{4}$ and $E_{3}, 13$ by $B_{4}$ and $A_{4}=E_{3}, 14$ by $D_{4}, 15$ by $C_{5}$ and $E_{4}, 16$ by $B_{5}$ and $E_{4}, 17$ by $D_{5}$ and $E_{4}, 18$ by $E_{5}$ and $E_{4}$.

For $n=11$, we try to find points $P_{1}, \cdots, P_{5}$ on one straight line, $P_{1}^{\prime}, \cdots, P_{5}^{\prime}$ such that $P_{k-1}^{\prime}, P_{k}$, and $P_{k+1}^{\prime}(k \bmod 5)$ are collinear and that $P_{k-2}^{\prime}, P_{k}$, and $P_{k+2}^{\prime}$ are collinear, and $P^{\prime \prime}$ such that $P_{k}, P_{k}^{\prime}$, and $P^{\prime \prime}$ are collinear. Let $P^{\prime \prime}=(0,0,1), P_{k}=\left(1,0, a_{k}\right), a_{0}=0$. We may suppose $P_{0}^{\prime}=(1,0, y)$, $P_{1}^{\prime}=\left(1, a_{1}, a_{1}\right), P_{2}^{\prime}\left(1, a_{2}, a_{2} x\right), P_{3}^{\prime}=\left(1, a_{3}, a_{3} x\right), P_{4}^{\prime}=\left(1, a_{4}, a_{4}\right)$; then all conditions of collinearity with $P^{\prime \prime}$ or $P_{0}$ are fulfilled. From the collinearity of $P_{1}^{\prime} P_{2} P_{3}^{\prime}$ and of $P_{2}^{\prime} P_{3} P_{4}^{\prime}$ there follows easily that $a_{2}^{-1}-a_{1}^{-1}=a_{4}^{-1}-a_{3}^{-1}$, and from the collinearity of $P_{0}^{\prime} P_{1} P_{2}^{\prime}$ and $P_{0}^{\prime} P_{4} P_{3}^{\prime}$ there follows $a_{1}^{-1}-a_{2}^{-1}=a_{4}^{-1}$ $-a_{3}^{-1}$; hence the characteristic of the field of coordinates must be 2 . Then all conditions are fulfilled provided that $a_{1}^{-2}+a_{2}^{-2}+a_{3}^{-2}=a_{1}^{-1} a_{2}^{-1}+a_{1}^{-1} a_{3}^{-1}+a_{2}^{-1} a_{3}^{-1}$, $x a_{3}\left(a_{1}+a_{2}\right)=a_{1}\left(a_{2}+a_{3}\right), y\left(a_{1}^{-1}+a_{3}^{-1}\right)=1$; these equations have solutions such that $0, a_{1}, a_{2}, a_{3}, a_{4}$ are all different, though possibly only after an extension of the field.

3.2. If $n$ points in a projective plane are on two straight lines, but not on one straight line, then there are at least three ordinary lines.

For if there are on either line at least two points $P_{i}$ different from the meeting-point, then there are at least four ordinary lines. Otherwise $n-1$ points are on one straight line, and either $n>3$ with $n-1$ ordinary lines or $n=3$ with three ordinary lines.

If $n$ points in a projective plane are not on a straight line, but $n-1$ of them are on two straight lines, then there are at least three ordinary lines.

For if there are on either line at least two points $P_{i}$ different from the meeting-point (otherwise we come back to the preceding statement), then either three connecting lines are ordinary, or two connecting lines and some line through the $n$th point, or two connecting lines and the two given lines.

3.3. The above $B_{m}(m>1)$ and $C_{m}(m>2)$ may be constructed formally for any $m$ (without imbedding in a projective plane) by considering points $P_{i}, P_{i}^{\prime}, P_{i}^{\prime \prime}, i=1, \cdots, m$, calling all $P_{i}$, all $P_{i}^{\prime}$; and all $P_{i}^{\prime \prime}$ collinear (in case $B_{m}$, with an additional point $\left.O\right)$, and defining $m^{2}$ collinear triples $P_{i} P_{j}^{\prime} P_{k}^{\prime \prime}$, 
for example, by the condition $i+j+k \equiv 0(\bmod m)$.

If $n$ points in a projective plane are on three straight lines, but not on one straight line, and if they do not form a set $B$ or $C$, then there are at least three ordinary lines.

Proof. By 3.2 we may assume that each line contains two points $P_{i}$ that are on neither of the other two lines. Then, if two of the given lines do not contain the same number of points $P_{i}$, there is, through every point $P_{i}$ on the third line, and not common to all three lines, at least one ordinary line. Hence there certainly exist three ordinary lines unless either all three lines contain the same number of points $P_{i}$, or they contain 2,2 , and $j>2$ points $P_{i}$ besides a common meeting-point. In the latter case there are at least $4 j-8 \geqq 4$ ordinary lines; in the former, if there is a point $P_{i}$ common to only two of the lines, or if there is a point $P_{i}$ common to all three lines and if the given points are not a set $B$, and finally if every $P_{i}$ belongs to only one of the lines and if the given points are not a set $C$, we easily establish the existence of at least three ordinary lines.

3.4. If $n$ points in a projective plane are not on one straight line, but if there is a straight line $q$ containing at least $n-5$ of the points, then either there are at least three ordinary lines, or $n=7$, the points being four points and their three diagonal points and the field of coordinates having the characteristic 2, where there is no ordinary line (case $B_{2}$ ).

For if the points $P_{i}$ are on three straight lines, apply the preceding proposition. Otherwise there are five points not on $q$, and no three of them are collinear. Through every point $P_{i}$ on $q$ there is an ordinary line, and for $n \leqq 7$, at least six of the ten lines connecting the five points are ordinary.

There follows:

If $n$ points in a projective plane are not on one straight line $(n=3,4,5,6,8)$, then there are at least three ordinary lines.

Seven points in a projective plane determine either at least three ordinary lines, or none; the latter happens only for seven collinear points and for points forming a set $B_{2}$.

We have also:

Nine points in a projective plane determine either at least three ordinary lines, or none; the latter happens only for nine collinear points and for points forming a set $C_{3}$.

For we may suppose that no straight line contains more than three of the given points. If there is an ordinary line $P_{1} P_{2}$, then through $P_{1}$ and $P_{2}$ there is a second and third ordinary line. If there is no ordinary line, let $P_{1}, P_{2}, P_{3}$ be collinear. It is easily seen that $P_{4}$ is collinear with two points $P_{5}$ and $P_{6}$, and $P_{7}$ with $P_{8}$ and $P_{9}$. Thus we can apply 3.3.

3.5. Note that there exist sets with exactly one or two ordinary lines. For example $A_{m}(m>2)$ less all points of one straight line but two is a set with one ordinary line; here $n$ is at least 11 . Similarly all points of $A_{m}(m>3)$, 
not on $P_{1} P_{2}$ and $P_{1} P_{3}$, together with $P_{1}, P_{2}, P_{3}$, form a set with two ordinary lines; $n$ is at least 15 .

3.6. Let $F$ be the field of coordinates. We may ask for conditions on $F$ such that $n$ points not on one straight line shall necessarily determine an ordinary line. So far one sufficient and two necessary conditions are known.

The sufficient condition for $F$ is that it be a (possibly non-Archimedean) ordered field. In this case the same proofs hold as for the field of real numbers.

One necessary condition is that the characteristic of $F$ be 0 . The configuration $A_{p}$ ( $p$ prime) occurs for characteristic $p$ (and only for it). Likewise $p \neq 0$ implies, for every $d>1$, the existence of a finite point set in $d$-space without ordinary hyperplanes (defined in 0.1 ).

The other necessary condition is that $F$ contain no roots of unity other than \pm 1 . (This implies again that $p$ is 0,2 , or 3.) For let $\{a\},\{b\},\{c\}$ be finite subsets of $F$ without 0 such that every product $a b$ is in $\{c\}$, every $b c$ in $\{a\}$, every $c a$ in $\{b\}$; a simple computation shows that one of them, say $\{a\}$, consists of the $m m$ th roots of 1 for some $m$, and either $\{b\}=\{c\}=\{a\}$ or $\{b\}=\{c\}=-\{a\}$. For $m>2$ the $3 m$ points $(0,-1, a),(b, 0,-1)$, $(-1, c, 0)$ form a set of the $C_{m}$ type and determine no ordinary line.

4. Existence of at least $n$ connecting lines, planes, or hyperplanes.

4.1. If $n$ points in the real projective plane are not on one straight line, then they determine at least $n$ connecting lines.

Proof. For $n \leqq 2$ there is nothing to prove. For $n>2$ suppose the theorem true for $n-1$. Among the $n$ given points consider one which is on an ordinary line. The other $n-1$ points are either on one straight line or determine at least $n-1$ connecting lines. In both cases the contention follows immediately.

By a remark at the end of 1.5 the theorem holds equally for points in real projective $d$-space, $d>2$.

Similarly one has:

The number of connecting lines is $n$ only if $n-1$ of the points are on one straight line. It can be $n+1$ only for $n=5$ and $n=6, n+2$ only for $n=4, n=6$, and $n=7$.

4.2. If $n$ points in real projective 3-space are not on one plane, then they determine at least $n$ connecting planes.

Proof. For $n \leqq 3$, there is nothing to prove. For $n>3$, suppose the theorem true for $n-1$. Among the $n$ given points, consider one which is on an ordinary plane such that the other given points on that plane are collinear. The other $n-1$ points are either on one plane or determine at least $n-1$ connecting planes. In the latter case the contention follows immediately; in the former apply 4.1 .

Again the theorem holds for points in real projective $d$-space, $d>3$.

Similarly:

The number of connecting planes is $n$ only if the points are on two straight 
lines. It can be $n+1$ only if $n-1$ points are on one plane and if, moreover, $n=6$ or $n=7$.

4.3. Using the abbreviations $\mathrm{T}_{d}$ and $\mathrm{U}_{d}$ of 0.1 we have:

If $\mathrm{U}_{d}$ and $\mathrm{T}_{d-1}$ hold then $\mathrm{T}_{d}$ holds, and the number of hyperplanes connecting $n$ points in projective d-space is exactly $n$ if and only if the points are, for odd $d$, on $(d+1) / 2$ straight lines, and for even $d$, all but one on $d / 2$ straight lines.

The proof of the first statement is as for 4.2. (It is true anyway, because of 4.5.) To prove the second part for $d=2 c-1$ note that for $c$ straight lines $p_{1}, \cdots, p_{c}$, with $n_{i}>1$ points $P_{i 1}, \cdots, P_{i n_{i}}$ on $p_{i}$ such that all these points are not on one hyperplane, every point $P_{i k}$ together with the lines $p_{j}, j \neq i$, determines a hyperplane $\pi_{i k}$; hence the number of connecting hyperplanes is the same as that of the given points. Now if a new point $P_{l}$ is neither on $\pi_{11}$ nor on $\pi_{21}$, then $P_{l}, P_{11}, P_{21}, p_{3}, \cdots, p_{d}$ determine a new hyperplane, different from any other similarly defined new hyperplane. Hence the number of new hyperplanes can be exactly one only if either $P_{l}$ is on every $\pi_{i k}, i \neq i_{0}$, whence $P_{l}$ is on $p_{i_{0}}$, or $P_{l}$ is on every $\pi_{i k}$ except say $\pi_{11}$ and $\pi_{21}$, whence $n_{1}=n_{2}=2$, $P_{l}$ on $P_{12} P_{22}$; in this case take $P_{11} P_{21}=p_{1}^{\prime}, P_{12} P_{22}=p_{2}^{\prime}$. The proof for $d=2 c$, $c>1$, is exactly "on the same lines," with an additional point $P_{0}$ and hyperplane $\pi_{0}=\left(p_{1} \cdots p_{c}\right)$.

4.4. If $n$ points $P_{i}$ in a projective plane are not on one straight line, then they determine at least $n$ connecting lines.

For suppose there are $m$ connecting lines $p_{1}, \cdots, p_{m}, 1<m \leqq n$, with $n_{k}+1$ given points on $p_{k}, n-2 \geqq n_{1} \geqq n_{2} \geqq \cdots \geqq n_{m} \geqq 1$. Besides $p_{1}$ there are at least $\left(n-n_{1}-1\right) / n_{2}$ connecting lines through every $P_{i}$ on $p_{1}$, whence $m \geqq\left(n-n_{1}-1\right)\left(n_{1}+1\right) / n_{2}+1$; by $n \geqq m$ we have

$$
n_{1}\left(n_{1}+1\right) \geqq(n-1)\left(n_{1}+1-n_{2}\right) .
$$

Hence if $n_{2}=1$, then $n_{1}+1 \geqq n-1 ; n-1$ points $P_{i}$ are on one straight line and $m=n$. For $n_{2}>1$ let $P_{1}$ be a given point on $p_{1}$ and if possible also on $p_{2}$. The other points $P_{i}$ on $p_{1}$ and $p_{2}$ are connected by at least $n_{1} n_{2}$ straight lines. Taking into account, as before, the connecting lines through $P_{1}$, we have $n \geqq\left(n-n_{1}-1\right) / n_{2}+n_{1} n_{2}+1$, that is, $n\left(n_{2}-1\right) \geqq n_{1} n_{2}^{2}+n_{2}-n_{1}-1$ or $n \geqq n_{1}\left(n_{2}+1\right)+1$. By $(1), n_{1}+1 \geqq\left(n_{2}+1\right)\left(n_{1}+1-n_{2}\right)$ or $n_{2} \geqq n_{1}$, whence $n_{1}$ $=n_{2}, n \geqq n_{1}^{2}+n_{1}+1$. Again by (1), $n \leqq n_{1}^{2}+n_{1}+1$, whence

$$
n=n_{1}^{2}+n_{1}+1 \text {. }
$$

Now let $n_{1}=\cdots=n_{f}>n_{f+1}$. Of the $C_{n, 2}$ pairs of points $P_{i}$, there are $C_{n, 2}-C_{n_{1}+1,2} f$ pairs that determine a straight line different from $p_{1}, \cdots, p_{f}$; hence the number of these lines is at least $\left(C_{n, 2}-C_{n_{1}+1,2} f\right) / C_{n_{1}, 2}$, whence $n \geqq m \geqq f+\left(C_{n, 2}-C_{n_{1}+1,2} f\right) / C_{n_{1}, 2}$. There follows $(n-f) n_{1}\left(n_{1}-1\right) \geqq n(n-1)$ $-f n_{1}\left(n_{1}+1\right)$ or, by $(2), 2 f n_{1} \geqq 2 n n_{1}, n \leqq f$. By $f \leqq m \leqq n$ we have $m=n$ as desired. 
Since $f=m$, there are $n_{1}+1$ points $P_{i}$ on every $p_{k}$. The meeting-point of any two $p_{k}$ is a $P_{i}$, since otherwise $m>\left(n_{1}+1\right)^{2}=m+n_{1}$. The $n$ given points form, therefore, in this case themselves a whole projective plane, and if this plane is Desarguesian, then $m_{1}$ is a power of the characteristic of the field of coordinates.

Another proof is as for the following generalization.

4.5. $\mathrm{T}_{d}$. If $n$ points in a projective $d$-space are not on one hyperplane, then they determine at least $n$ connecting hyperplanes.

$\mathrm{T}_{1}$ is trivial. By $\mathrm{T}_{d}-1$ we have $\bar{h} \leqq \bar{p}$, where $\bar{h}$ is the number of given points on one of the connecting hyperplanes $h$ and $p$ the number of connecting hyperplanes through one of the given points $p$ that is not on $h$. Now apply the lemma 4.6.

It follows by projection on $(m+1)$-space, possibly after extension of the field of coordinates, that $n$ points in a projective $d$-space that are not on one $m$-dimensional linear manifold determine at least $n$ such manifolds. In particular, $n$ points in real spherical $d$-space that are not on one $m$-dimensional sphere determine at least $n$ such spheres.

4.6. $\mathrm{L}_{e}$. If $n$ not necessarily different nonvoid subsets of a set of e elements have the property that for every given subset $p$ and element $h$ not in $p$ we have $\bar{h} \leqq \overline{\text { ( }} \bar{h}$ being the number of given subsets containing $h$ and $\bar{p}$ the number of elements of $p$ ) and if no element belongs to all the subsets, then $e \geqq n$.

$\mathrm{L}_{1}$ is void, $\mathrm{L}_{2}$ trivial. If $n>e>2$ we show that there exists a proper subsystem $P=\left(p_{1}, \cdots, p_{e}\right)$ of the given system of subsets such that $h_{i}$ is not in $p_{i}, i=1, \cdots, e$. Then we would have $\bar{h}_{i} \leqq p_{i}, \sum \bar{h}_{i} \leqq \sum \bar{p}_{i}$, while $\sum \bar{h}_{i}$ equals the sum of all $\bar{p}$. In order to see that $P$ ought to exist, we make use of the well known theorem (Frobenius and others) that an $n$-row determinant of zeros and indeterminates is zero if and only if the matrix contains a rectangle of $j \cdot k$ zeros, $j+k=n+1$. We apply this to a matrix $\left(a_{\mu \nu}\right), \mu, \nu=1, \cdots, n$, where $a_{\mu \nu}=0$ if and only if $\mu \leqq e$ and $h_{\mu}$ is in $p_{\nu}$ and see that $P$ exists except if $k$ sets $p$ contain the same $j$ elements $h_{1}, \cdots, h_{j}, j+k=n+1$. Now for $j=1$, $h_{1}$ would belong to all the given sets. For $1<j<e$ we have $j \leqq n-2, k \geqq 3$. Omit $k-2$ from among the above $k$ sets, and in each of the remaining $n-k+2$ sets delete all elements other than $h_{1}, \cdots, h_{j}$. The system obtained fulfills the conditions of $\mathrm{L}_{j}$ : if one of its subsets were void all would be void, since $k-2 \geqq e-j$ if $e \leqq n-1$; and $\bar{h} \leqq p$ implies $\bar{h}-(k-2) \leqq p-(e-j)$. Hence $j \geqq n$ $-(k-2)$ which is not so. Finally, if $j=e$, we omit one of the $k$ sets, delete one of the elements, and apply $\mathrm{L}_{e-1}$.

5. Remark on complete configurations. A configuration $\left(a_{b}, c_{d}\right)$ of $a$ points and $c$ straight lines in a projective plane, any point of which is on $b>1$ lines and on any line of which there are $d>1$ points (whence $a b=c d$ ), is line-complete if it contains every straight line through two of its points; the dual configuration is point-complete. Since among $C_{a, 2}$ pairs of points, $c C_{d, 2}$ are connected by lines of the configuration, the condition for line-complete- 
ness is $c=c_{a, 2} / C_{d, 2}$. By 1.1 we have:

In the real plane the only line-complete configuration is the trivial configuration $a_{a-1},\left(C_{a, 2}\right)_{2}$. Only for $a=3$ is this configuration also point-complete.

This contains a simple, purely geometrical proof of the nonrealizability of $c_{3}=\left(9_{4}, 12_{3}\right)$ (the inflexions of a cubic) in the real plane.

To find the combinatorial formula of all line-complete configurations note that $b=(a-1) /(d-1)$ whence $a=1+b(d-1), c=(1+b(d-1)) b / d$; putting $e=b^{2}-c=b(b-1) / d$, the formula becomes $1+b(d-1)_{b}, b^{2}-e_{d}$. The first cases with $d>2$, ordered by ascending $a b=c d$, are

$$
\begin{aligned}
& 1: 7_{3}, 7_{3} ; 2: 9_{4}, 12_{3} ; 3: 16_{3}, 8_{6} ; 4: 13_{4}, 13_{4} ; 5: 13_{6}, 26_{3} ; \\
& 6: 16_{5}, 20_{4} ; 7: 21_{4}, 14_{6} ; 8: 15_{7}, 35_{3} ; 9: 21_{5}, 21_{5} ; 10: 25_{6}, 30_{5} .
\end{aligned}
$$

Of these, 3 and 7 are not realizable in any projective plane, because of $c<a$ and 4.4 .

If the configuration shall also be point-complete then $b^{2}-b(b-1) / d=1$ $+d(b-1)$; subtracting with $b$ and $d$ interchanged we have $(b-d)(b+d)(b-1)$ $\cdot(d-1)=0$, whence $b=d$. We obtain the configurations $A_{b-1}=\left(b^{2}-b+1_{b}\right.$, $\left.b^{2}-b+1_{b}\right)$ of 3.1 and 4.4 , including the above cases $1,4,9$. From such "finite projective planes," finite affine planes such as 2, 6, 10 are obtained by deleting one line and its points. Case 8 can be realized by projecting a finite projective 3-space.

Hebrew University, JERUSALEM, ISRAEL.

Boston College,

Boston, Mass. 